\title{
THz radiation from delta-doped GaAs
}

\author{
Birkedal, Dan; Keiding, S.R
}

Published in:

Proceedings of 5th European Quantum Electronics Conference

Publication date:

1994

Document Version

Publisher's PDF, also known as Version of record

Link back to DTU Orbit

Citation (APA):

Birkedal, D., \& Keiding, S. R. (1994). THz radiation from delta-doped GaAs. In Proceedings of 5th European Quantum Electronics Conference (pp. 116-116). IEEE.

\section{General rights}

Copyright and moral rights for the publications made accessible in the public portal are retained by the authors and/or other copyright owners and it is a condition of accessing publications that users recognise and abide by the legal requirements associated with these rights.

- Users may download and print one copy of any publication from the public portal for the purpose of private study or research.

- You may not further distribute the material or use it for any profit-making activity or commercial gain

- You may freely distribute the URL identifying the publication in the public portal

If you believe that this document breaches copyright please contact us providing details, and we will remove access to the work immediately and investigate your claim. 


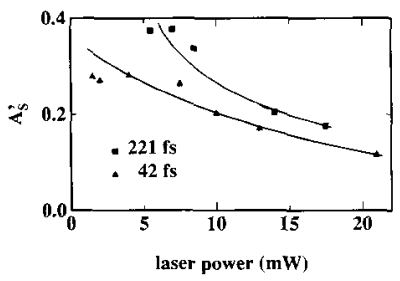

QWB5 Fig. 2. Power dependence of the normalised interface contribution A, measured at $-4-\mathrm{V}$ bias with a pulsewidth of $221 \mathrm{fs}$ and $42 \mathrm{fs}$. The points are connected to guide the eye.

open circles present data at $0-\mathrm{V}$ bias, the dots at $-4 \mathrm{~V}$.

Figure 1a shows a very clear bias dependence that disappears from 19-mW excitation as shown in Fig 1b. The measured $I_{2 w}$ curves can be fitted to Eq. (1) ured $I_{2 w}$ curves can be fitted to Eq. (1)
very well with $A_{B}, A_{S}$ and $\phi$ as fitting very well with $A_{B}, A_{S}$ and $\phi$ as fitting
parameters. The bulk contribution $A_{B}$ appears to be constant and insensitive to the applied bias voltage. This means that the bulk susceptibility is not affected by a depletion layer or injected carriers. The norpletion layer or injected arriers. The normalised interface contribution $A_{S}=$
$A_{S} / A_{B}$, depends on the effective electric field at the SB interface. $A_{s}^{\prime}$ appears to be proportional to the square root of the applied reversed bias and the zero-biasbandbending, in accordance with the Schottky model. From Fig. $1 \mathrm{~b}$ it can be seen that the bias dependence of $A_{s}^{\prime}$ is much smaller for higher average laser powers. This power dependence can be explained by the effect of carrier injection. If generated holes reach the Au layer, the charges will be compensated to a certain extent and thus the effective electric field will decrease. This decrease of the field takes place on a femtosecond timescale. this means that the power dependence This means that the power dependence of $A_{S}^{\prime}$ also depends on the width of the
laser pulses. Figure 2 shows the power dependence of $\mathrm{A}_{s}^{\prime}$ measured at -4 -V bias with a pulsewidth of $221 \mathrm{fs}$ and $42 \mathrm{fs}$. Monte Carlo simulations performed to calculate this power and pulsewidth dependence are in qualitative agreement with these observations.

${ }^{*}$ Department of Physics, Eindhoven University

of Technology, P.O. Box 513,5600 MB

Eindhoven, The Netherlands

QWB6

0945

THz radiation from delta-doped GaAs

D. Birkedal, S. R. Keiding, ${ }^{*}$ Mikroelektronik Centret, Building 345 East, Technical University of Denmark DK-2800 Lyngby, Denmark

The emission of ultrafast $\mathrm{THz}$ pulses from semiconductor surfaces after femtosecond laser excitation was recently observed by Zhang and Auston. ${ }^{1}$ Photoex served by Zhang and Auston. Photoexcited carriers created near the surface are accelerated by the electric field present ing photo current radiates an electro magnetic pulse possessing frequency components in the THz-regime.

The presence of a delta-doped layer in the vicinity of the surface creates a very

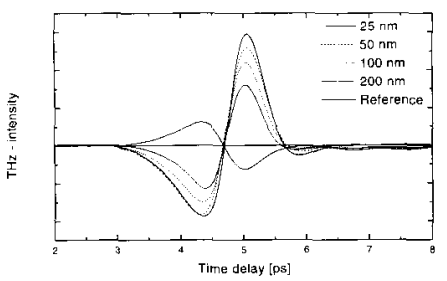

QWB6 Fig. 1. THz-pulse radiated from the 4 delta-doped samples and the LEC GaAs reference sample with the $\mathrm{Ti}$ sapphire laser operating at $830 \mathrm{~nm}$.

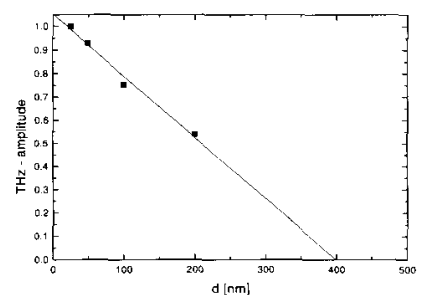

QWB6 Fig. 2. Rms values of the

QWB6 Fig. 2. Rms values of the THz-pulses emitted from the delta-
doped sample relative to the $d=25 \mathrm{~nm}$ sample as a function of delta-layer depth. The line represents a linear fit to the data.

large field at the surface. These characteristics of the delta-doped structures make them very interesting as sources of make them very interesting as sources of and the high mobility should make the emitted $\mathrm{THz}$ amplitude greater than that emitted from bulk GaAs surfaces.

The delta-doped GaAs samples used in this work were grown by molecular beam epitaxy on $\mathrm{Cr}$-doped semi-insulating GaAs substrates. The delta-doping was of $n$-type with $\mathrm{Si}$ as dopant. As reference samples we used a semi-insulating liquidencapsulated Czochralski (LEC) GaAs sample. The fields present on both sides of the delta-doped layer are measured by photoreflectance.

The experimental setup used in the $\mathrm{THz}$ experiments is described in Ref. 2 . $\mathrm{THz}$ signals radiated from the deltadoped samples upon laser pumping at $840 \mathrm{~nm}$ are shown in Fig. 1. The curves are labelled to indicate the type of sample. Also shown in a pulse radiated from ple. Also shown in a pulse radiated from the LEC GaAs reference sample. It is observed that the THz-pulses from the
delta-doped samples show the opposite polarity of the THz-pulse emitted by the LEC GaAs sample. We also observe a decrease of the pulse amplitude as the distance from the surface to the delta-doped layer is increased.

The sign of the electric field radiated from the surface, and correspondingly the direction of the photo-current can be determined from the measured pulses by calibrating the response of the detection system with a biased THz-antenna. We find that the observed polarity of the pulses radiated from the delta-doped pulses radiated from the delta-doped
samples corresponds to a net current di- rected toward the substrate. This is consistent with the majority of the $\mathrm{THz}$ sig nal arising from the substrate side of the delta-doped layer.

We show in Fig. 2 the rms values of the THz-pulses emitted from the four delta-doped samples as a function of the position of the delta-doped layer. The straight line is a fit to the data and is explained in the following way: The source current responsible for the radiated $\mathrm{THz}_{\mathrm{z}}$ pulse originates from both sides of the delta-doped layer. Hence, the surface and substrate side contributions have opposite sign; the total $\mathrm{THz}$ signal is the sum of these contributions. As the distance from the surface to the delta-doped layer is increased the source current from the surface side is increased and the total THz-power is decreased.

* Fysisk Institut, Odense Universitet

Campusvej 55, DK-5230 Odense M, Denmark

1. X. C. Zhang, D. H. Auston, J. Appl. Phys. 71, 326 (1992)

2. J. E. Pedersen, I. Balslev, J. M. Hvam, S. R. Keiding, Appl. Phys. Lett. 62, 1392 (1992).

QWB7 (Invited) 1000 Ultrafast coherent solid-state phenomena

T. Dekorsy, T. Pfeifer, G. C. Cho, P. Leisching, H. G. Roskos, H. Kurz, Institute of Semiconductor Electronics, RWTH Aachen, 52056 Aachen, Germany

The progress of laser sources with subpicosecond pulse widths allows the preparation of coherent states in solid-state material and to study their dephasing in time domain Of particular interest is the investigation of coherent phenomena in semiconductors and semiconductor heterostructures, revealing information on coherent electronic and vibronic states in these materials. Details on the generation and the dephasing of coherently excited phonons and electrons can be directly obtained in the time domain by pumpprobe spectroscopy. Amplitude and probe spectroscopy. Amplitude and phase of this motion can be simultane-
ously traced by electrooptic measuring techniques. Once the coherent state is prepared, it can be accurately controlled by further phase-sensitive excitation of the system.

We review the results obtained in compound semiconductors and semiconductor heterostructures with a detection method that is based on the time-resolved optical modulation due to the Pockels-effect. ${ }^{2}$ This technique has a high sensitivity towards internal polarizations sensitivity towards internal polarizations associated with coherently excited LOphonons and coherent electronic wave functions, e.g., Bloch oscillations in superlattices.

The time-resolved electrooptic detection of coherent longitudinal optical (LO) phonons in GaAs, which are launched by ultrafast screening of surface-spacecharge fields, allows to display the decharge fields, allows to display the dephasing in time domain with high accuracy and time resolution. Thus on a subpicosecond timescale directly for the first time. At low excitation levels, LO-phonons decay by the well known anharmonic coupling. With increasing anharmonic coupling. With increasing 